\title{
Design and simulation of circularly polarized pentagonal-shaped microstrip patch antenna at RFID frequency $2.4 \mathrm{GHz}$
}

\author{
Bondili Kohitha Bai, Bhupendra singh* and vinod shokeen* \\ Department of electronics and communication Engineering, ASET, Amity \\ University, Noida, India. \\ kohitha@gmail.com.
}

\begin{abstract}
In wireless communication system antennas play an inherent role. In terms of geometrical shapes and implementations microstrip patch antennas are versatile in nature. Characteristics like low gain and smaller bandwidth make single microstrip more popular. Printed type of antenna which consists of a radiating patch on one side of a dielectric substrate and ground plane on the other side is a microstrip patch antenna. The intension of exploiting design is to implement a circularly polarized pentagonal-shaped microstrip patch antenna with a dielectric constant of 2.33, for a dielectric substrate. The antenna is designed and simulated using IE3D electromagnetic simulator. Circularly polarized pentagonal-shaped microstrip patch antenna has good CP axial ratio bandwidth and minimum reflection coefficient. The aimed antenna designed for WLAN applications at RFID frequency of $2.4 \mathrm{GHz}$ in ISM band. The simulation outcomes shows that the designed $C P$ pentagonal shaped microstrip patch antenna gives axial ratio of 0.6023 at $2.38 \mathrm{GHz}$ and $\mathrm{CP}$ axial ratio bandwidth of $36 \mathrm{MHz}$ with $1.5 \%$.
\end{abstract}

\section{KEYWORDS}

Microstrip patch antenna, circular polarization, IE3D software.

\section{INTRODUCTION}

Microstrip patch antenna is a printed type of antenna with a dielectric substrate inserted in between a ground plane and a patch. Microstrip patch antenna is a narrow band wide beam antenna fabricated by etching the antenna element pattern in metal trace bonded to an insulating dielectric substrate with a continuous metal layer bonded to the opposite side of the substrate which forms a ground plane. The size of the patch antenna directly related to the resonant frequency. The microstrip patch antennas are simple to fabricate and easy to modify and customize. The metallic patch can take different types of configurations such as rectangular patches, circular patches, triangular patches and pentagonal patches.

Natarajan Meghanathan, et al. (Eds): ITCS, SIP, JSE-2012, CS \& IT 04, pp. 01-10, 2012.

(C) CS \& IT-CSCP 2012

DOI : $10.5121 /$ csit.2012.2101 
The microstrip antennas are low profile antennas, comformable to planar and non-planar surfaces, simple and inexpensive to fabricate using modern printed technology. These antennas are mechanically robust when mounted on rigid surfaces, and are versatile in terms of resonant frequency, polarization, radiation pattern and impedance. These antennas can be mounted on the surface of high-performance aircraft, spacecraft, satellites, missiles and also handheld mobile telephones. Microstrip patch antennas are also relatively inexpensive to manufacture and design because of the simple 2-dimensional physical geometry. They are usually employed at UHF, ISM band and higher frequencies because the size of the antenna is directly tied to the wavelength at the resonance frequency.

The advantage inherent in patch antennas is the ability to have polarization diversity. It means patch antennas can easily be designed to have Vertical, Horizontal, Right Hand Circular (RHCP) or Left Hand Circular (LHCP) Polarizations, using multiple feed points, or single feed point with asymmetric patch structures. This unique property allows patch antennas to be used in many types of communications links that may have varied requirements.

Several microstrip patch antennas are proposed for many wireless applications[1]. Most of the research is focused on rectangular microstrip patch antenna since it is simple to design. This antenna gives both linear and circular polarization. However, rectangular patch antenna needs two feeds to get circular polarization [2].

The project deals with the design and simulation of circularly polarized antenna which can be easily implemented by properly cutting a small sized section from a side of the equilateralpentagon patch in which the fundamental resonant mode of the equilateral - pentagon microstrip antenna is split into two near-degenerate orthogonal modes with equal amplitudes and a 90' phase difference [3]. The pentagonal-shaped microstrip patch antenna can be designed with only one probe feed to get circular polarization and it can be used for many wireless applications at different frequencies [4]. The project deal with an antenna which is designed for WLAN application at RFID frequency $2.4 \mathrm{GHz}$ in Industrial scientific medical (ISM) band.

IE3D simulator is used to design and simulate the proposed microstrip patch antenna. IE3D is an integrated full-wave electromagnetic simulation and optimization package for the analysis and design of 3D and planar microwave circuits, MMIC, RFID, RFIs, antennas, digital circuits and printed circuit boards.

\section{MICRO STRIP PATCH ANTENNA}

Microstrip antenna is commonly used at frequencies from 1 to $100 \mathrm{GHz}$ and at frequencies below ultra high frequency, UHF microstrip patch become exceptionally large. The radiating patch can be design in various shapes according to the desired characteristics like $\mathrm{H}$-shape micro strip antenna [5].

The first step is to choose the suitable substrate. In most cases, considerations in substrate characteristics involved the dielectric constant and loss tangent and their variation with temperature and frequency, dimensional stability with processing, homogeneity and isotropicity. The surface of the substrate has to be smooth to reduce losses and adhere well to the metal used. Substrate thickness and permittivity determine the electrical characteristics of the antenna. Thicker substrate will increase the bandwidth but it will cause the surface waves to propagate and 
spurious coupling will happen. This problem however, can be reduced or avoided by using a suitably low permittivity substrate. Five categories of dielectric material that can be used as substrates [3].

\subsection{Pentagonal -shaped Microstrip Patch antenna}

Circular polarization is theoretically possible from a microstrip antenna excited by a single feed if two spatially orthogonal modes are excited in phase quadrature. This can be achieved in a pentagonal patch as shown in Figure 1 below.

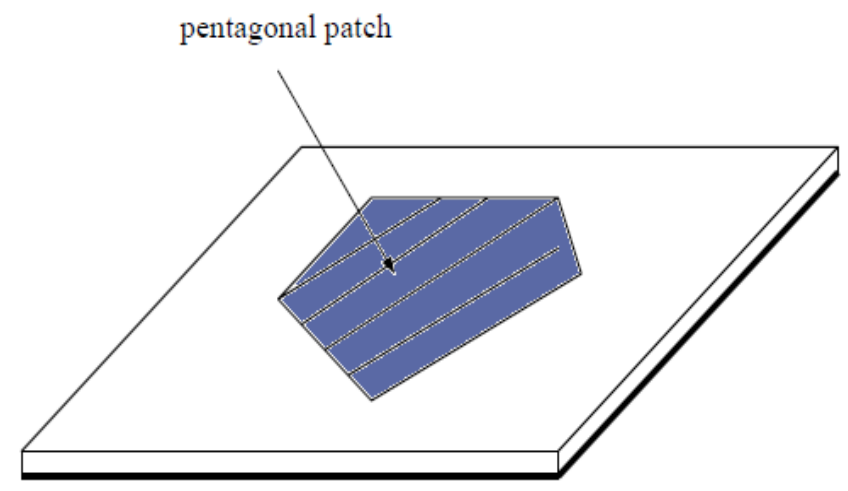

Figure 1 Geometry of a pentagonal patch.

Pentagonal microstrip patch antenna supports both linear and circular polarization. It gives better performance compared to the rectangular patch antenna. The pentagonal patch antenna gives circular polarization with only one feed where as rectangular patch antenna requires multiple feeds to get circular polarization [6]. The pentagonal patch antenna can also use multiple feeds. And this type of antenna with multiple feeds can also give multiband operations.

In single-fed CP pentagonal patch antenna, the generated mode usually excited in an electrically thin cavity region of the microstrip antenna. Accordingly, the operation principle of this antenna is based on the fact that the generated mode can be separated into two orthogonal modes [7].

\section{Design procedure}

A single-fed circularly polarized microstrip antenna designed as follows.

The patch geometries and probe locations are optimized for best axial ratio results on infinite ground plane and then transported to finite ground planes of different shapes for simulation on FIDELITY. The pentagonal patch geometry on different ground plane shapes is shown in Fig 2. The finite pentagonal ground plane was designed with the largest side equaling hand other sides with same ratios as the radiating pentagonal patch [3]. 


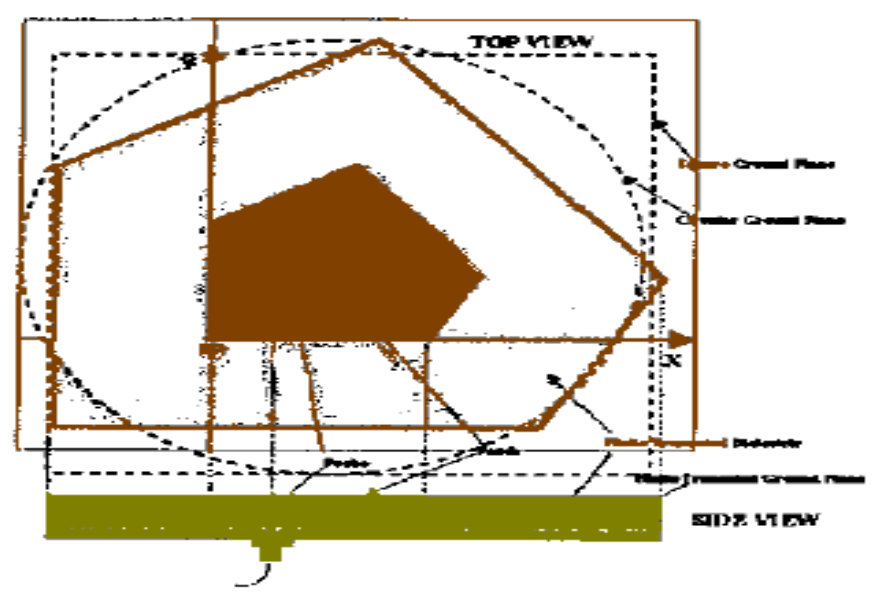

Figure 2 Probe-fed, circularly polarized, pentagonal patch antenna.

\subsection{Design and simulation}

The circularly polarized antenna which can be easily implemented by properly cutting a small sized section $(\Delta \mathrm{L})$ from a side of the equilateral-pentagon patch in which the fundamental resonant mode of the equilateral - pentagon microstrip antenna and it is split into two neardegenerate orthogonal modes with equal amplitudes and a 90' phase difference [8]. The designed antenna which is operating at $2.4 \mathrm{GHz}$ with circular polarization has many advantages compared to the other microstrip patch antennas [9].

This can be shown in figure 3

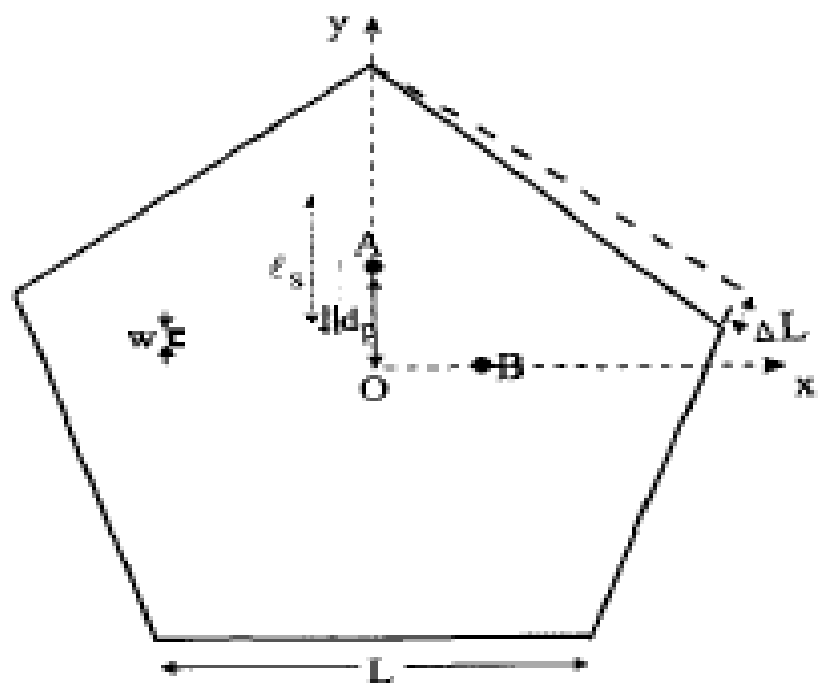

Figure 3 Equilqteral pentagon with $\Delta \mathbf{L}$. 


\subsection{Tool}

IE3D is an full-wave electromagnetic simulation and optimization package. It is a full-wave electromagnetic solver. It solves maxwells equations which govern the macro electromagnetic phenomenon. There is no much assumption involved except the numerical nature of the method. Therefore the solution is accurate. The original maxwells equations are in differential form and the solutions of the equations are the electric field and magnetic field in the whole space.The solution involves many unknowns. Instead, the IE3D solves the maxwells equations in an integral form through the use of grren's functions.

IE3D mainly focuses on general planar and 3D metallic structures in layered dielectric environments. It can also model 3D dielectric structures such as patch antennas with finite substrates and dielectric resonator antennas.

\subsection{The configuration of IE3D's main layout editor}

MGRID serves two purposes.

- Preprocessor as layout editor.

- Postprocessor for s-parameter and current distribution visualization, pattern calculation, near field calculaion and visualization, lumped element extraction.

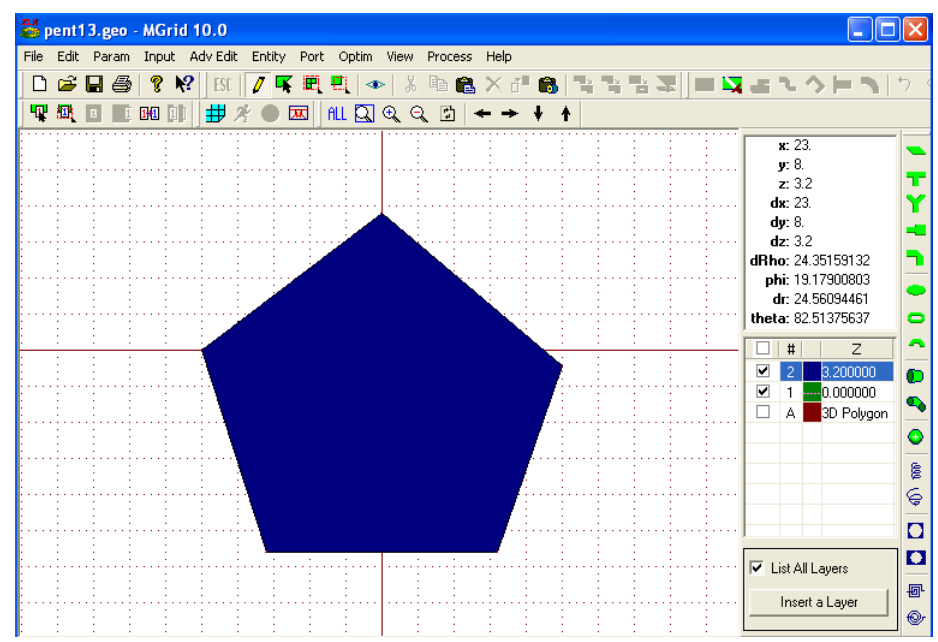

Figure 4 Basic configuration of the MGRID window.

\subsection{Basic parameters}

The basic parameters include 6 groups of parameters.

- Comment: The comment for the structure.

- Length: The length unit and minimum length for the structure.

- Layouts and Grids: the parameters for the uniform grid system for the layout editing. 
- Enclosure: the walls for enclosures used in the structure.

- Meshing parameters: the parameters control the meshing of the geometry.

- Substrate layers: The parameters of substrate and infinite ground planes.

- Metallic strip types: The parameters for different types of metallic strips used in structure.

- Dielectric type: for 3D dielectrics and finite size substrates.

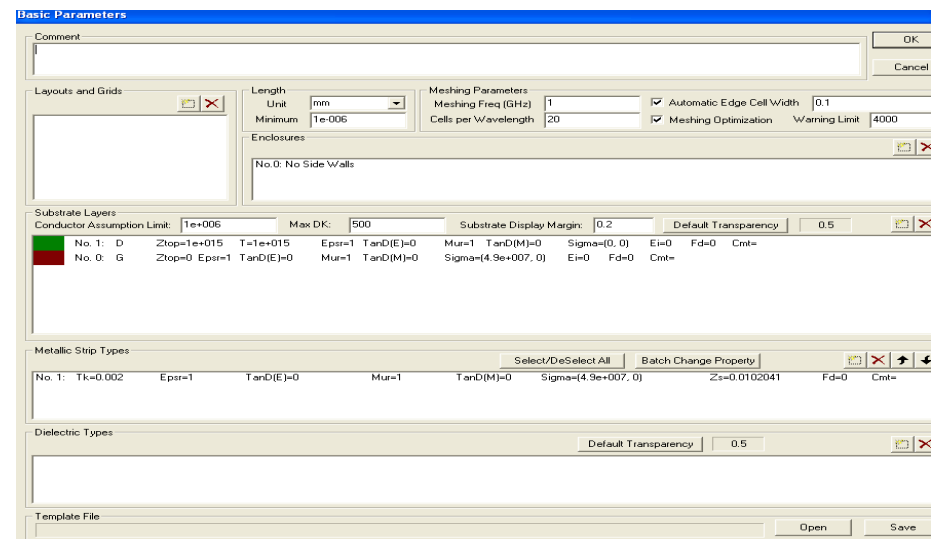

Figure 5 Basic parameters Window.

\subsection{Electromagnetic simulation}

Select process ->simulate command. MGRID prompts for the simulation setup dialog as shown in figure below.

There are two types of files involved in an IE3D simulation.

- .GEO files storing the geometry description.

- .SIM files storing the simulation control parameters such as frequency points, matrix solver.

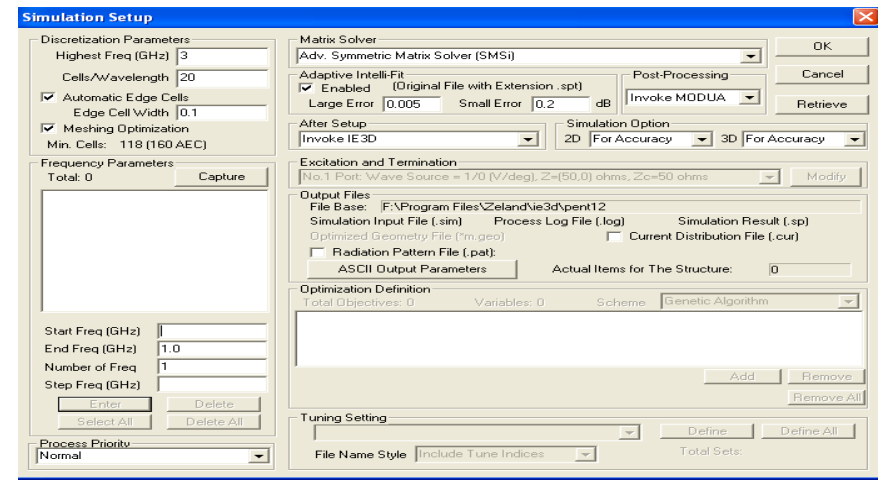

Figure 6 Simulation setup window. 
After entering the required data we press OK and then simulation begins.IE3D dialog will display the intermediate simulation information.

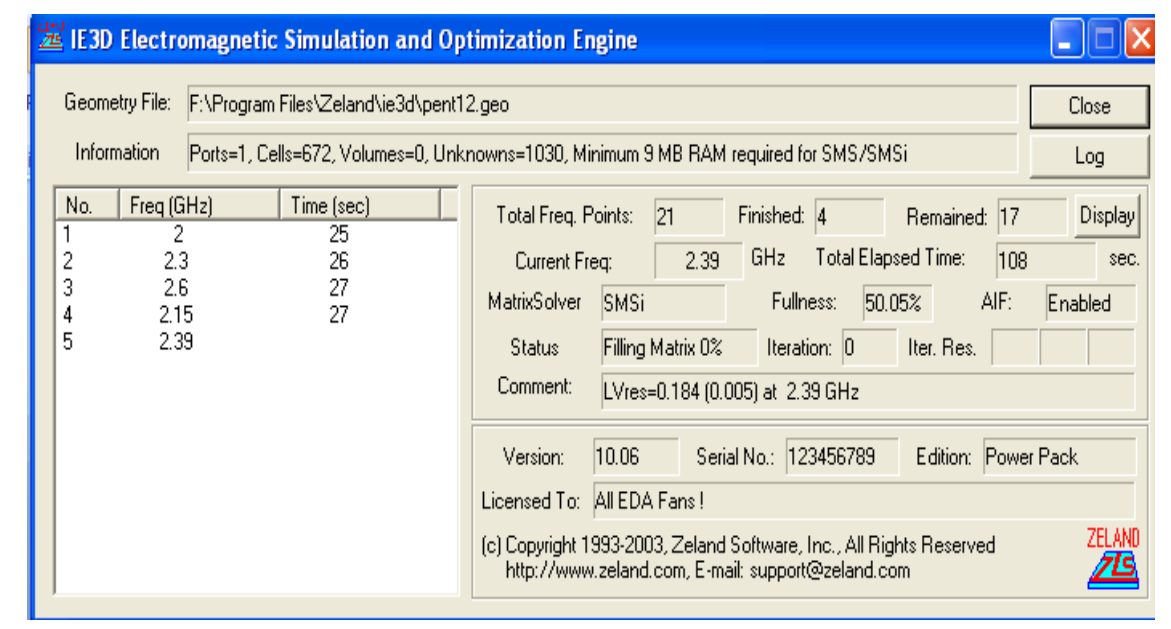

Figure 7 Electromagnetic simulation and optimization.

\subsection{Radiation pattern visualization}

When we check "Radiation pattern file" in the simulation setup dialog, MGRID will invoke IE3D to simulate the structure and perform pattern calculation automatically.

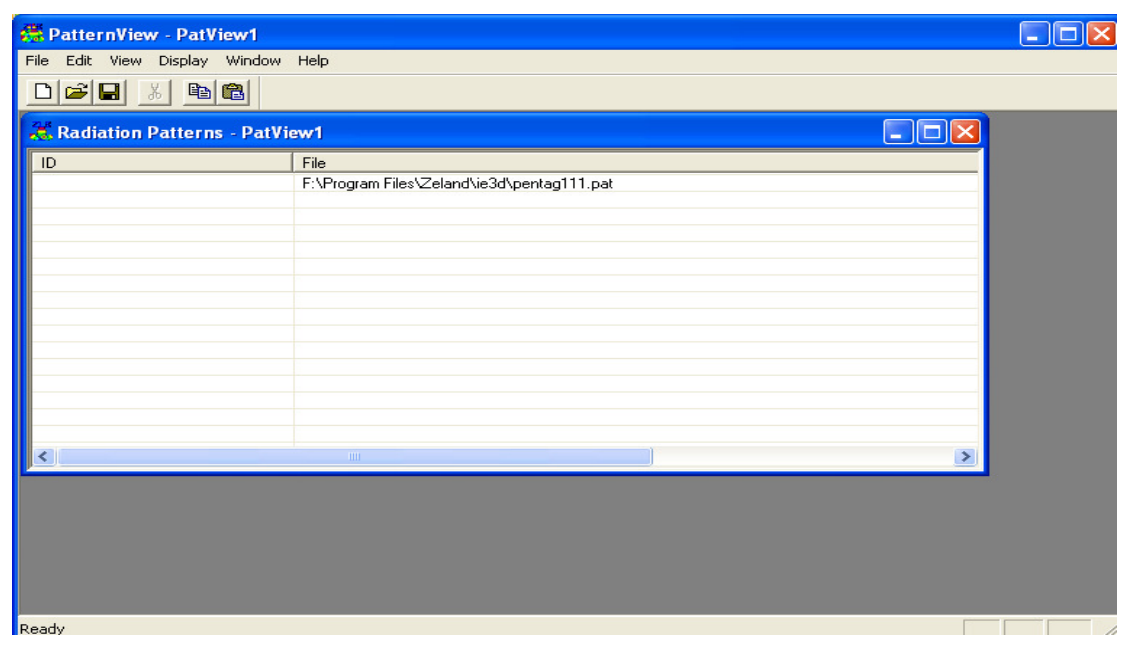

Figure 8 pattern view.

\section{Design of Pentagonal patch}

The Substrate that considered here is RT Duroid which is having dielectric constant $\varepsilon r=$ 2.33 with Thickness $\mathrm{h}=3.2 \mathrm{~mm}$, Loss tangent $=0.0018$ and pentagonal patch design with feed point at $(8.8,-9.5)$. 


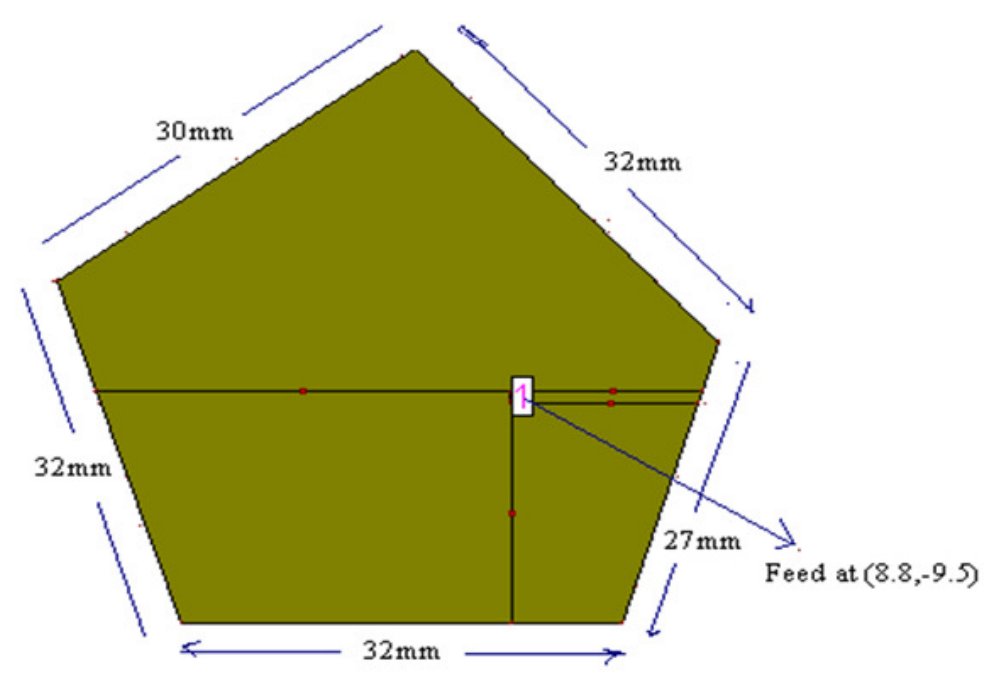

Figure 9 Geometry of the pentagonal patch.

\section{Simulation results}

\subsection{Reflection coefficient (S11 in dB)}

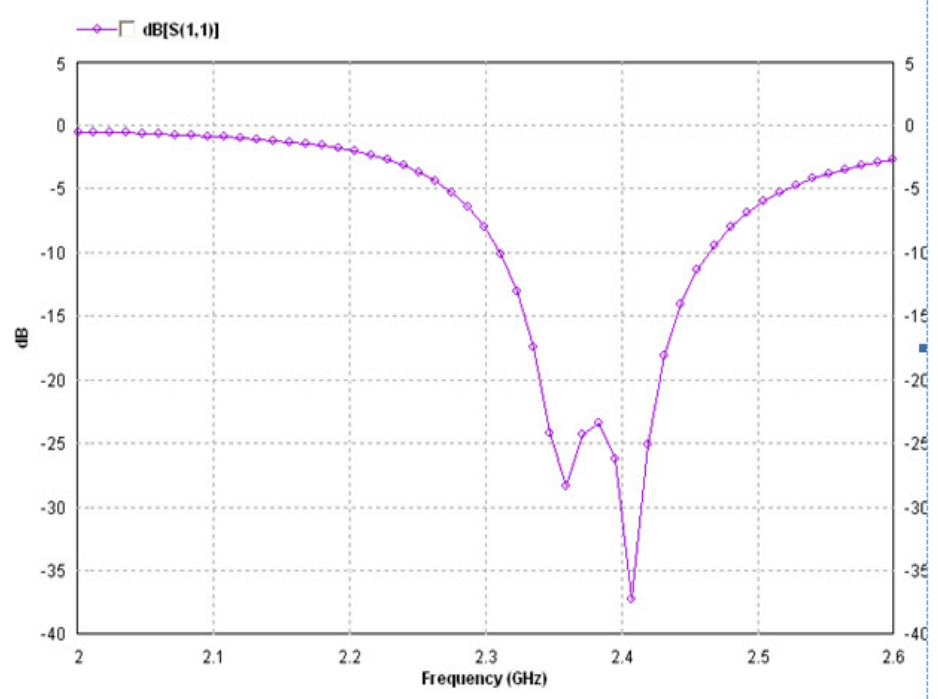

Figure 10 Reflection coefficient. 
5.2. Smith Chart

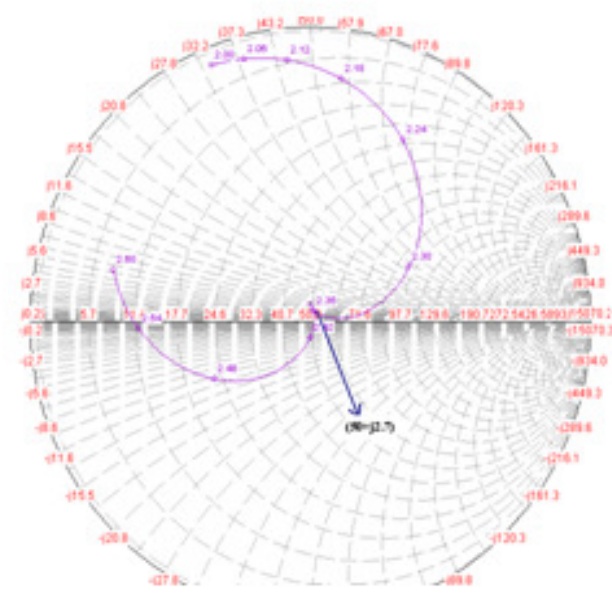

Figure 11 Smith chart.

\subsection{Axial ratio}

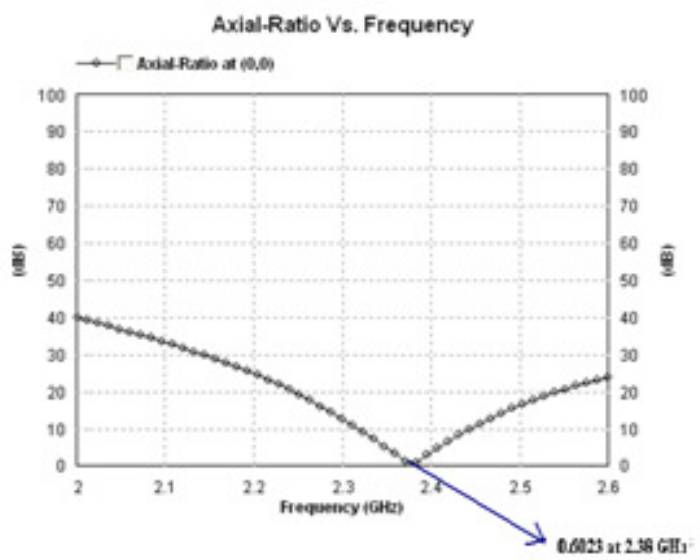

Figure 13 Axial ratio vs frequency.

\subsection{Radiation pattern}

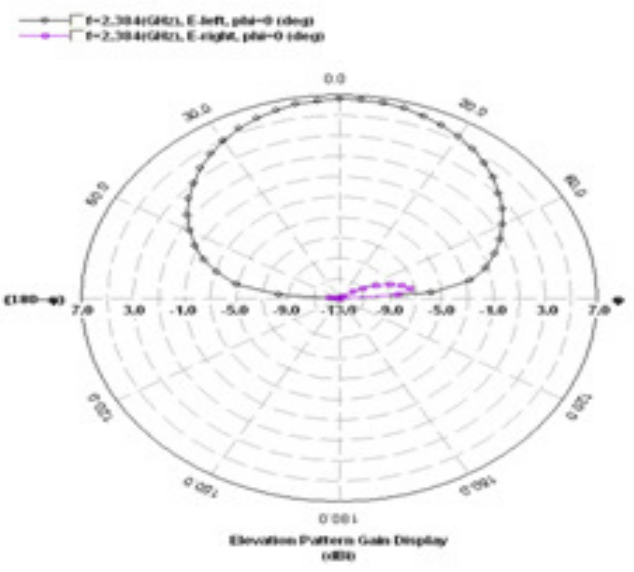

Figure 12 Radiation pattem.

\subsection{Gain}

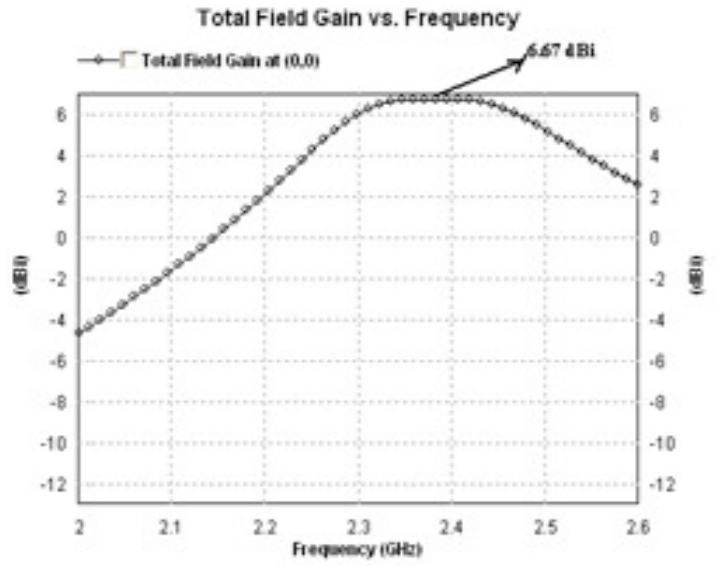

Figure 14 Gain vs Frequency

\section{Conclusions}

The circularly polarized pentagonal-shaped microstrip patch antenna at RFID frequency $2.4 \mathrm{GHz}$ has been designed and simulated using IE3D full-wave electromagnetic simulator. The proposed antenna gives an improved $\mathrm{CP}$ axial ratio bandwidth and minimum reflection coefficient. This antenna can be used in WLAN applications. The designed antenna which is operating at $2.4 \mathrm{GHz}$ with circular polarization has many advantages compared to the other microstrip patch antennas. Simulation results shows that the designed antenna is capable of generating circular polarization (CP) with axial ratio of 0.6023 and $\mathrm{CP}$ axial ratio bandwidth of $1.5 \%$. And the antenna designed 
gives $\mathrm{CP}$ axial ratio-3 $\mathrm{dB}$ bandwidth of $36 \mathrm{MHz}$. The designed pentagon shaped microstrip patch antenna gives reflection coefficient i.e.; S11 (dB) $-31 \mathrm{~dB}$ which is minimum at resonant frequency of $2.38 \mathrm{GHz}$. Designed antenna gives $6.7 \mathrm{dBi}$ gain at $2.38 \mathrm{GHz}$ with good circular polarization.

\section{REFERENCE}

[1] Secmen, M. and Hizal,"A. An inverted L-shape fed microstrip patch antenna for mobile communication "International Symposium on Personal Indoor and Mobile Radio Communications (PIMRC), 2010 IEEE, on page(s): $1102-1106$.

[2] Wen-Shyang Chen, Kin-Lu Wong and Jeen-Sheen Row , "Superstrate loading effects on the circular polarization and cross polarization characteristics of a rectangular microstrip patch Antenna" IEEE Transactions on Antennas and Propagation, on page(s): 260 - 264.

[3] Wen-Shyang Chen and Horng-Dean Chen- "Compact circularly polarized pentagonal shaped microstrip antenna with bent losses" Antenna and propagation society international symposium ,2001 IEEE ,Page(s):424-426,vol.3

[4] Yuh-Yih Lu,Shih-Chiang Wei,Hsiang-Cheh Huang, Design of RFID Antenna for $2.45 \mathrm{GHz}$ Applications, Fourth International Conference on Innovative Computing, Information and Control (ICICIC), 2009, On page(s): $601-604$.

[5] Performance of H-shaped microstrip antenna using IE3D, Kant Singh.R,Dhubkarya.P, D.C. Emerging Trends in Electronic and Photonic Devices \& Systems, 2009. International Conference on. 2009 ,On page(s): $364-366$.

[6] A novel wide beam circular polarization antenna - microstrip-dielectric antenna, He Haidan, Proceedings in Microwave and Millimeter Wave Technology, 2002. On page(s): 381 - 384.

[7] Wideband microstrip antenna for land based vehicles- Research article by Associate Professor Ir. Dr. Wan Khairuddin Ali, RESEARCH VOT: 74038.

[8] Natarajan and D. Chatterjee-'Effects of Ground Plane Shape on Performance of Probe-Fed, Circularly Polarized, Pentagonal Patch Antenna" Antennas and propagation Society International Symposium, 2003. IEEE, Page(s): 720 - 723.

[9] Rashid A. Saeed and Sabira Khatun-"Design of Microstrip Antenna for WLAN"- Journal of applied Sciences 5 (1): 47-51, 2005 ISSN 1607-8926 @ 2005 Asian Network for Scientific Information. 